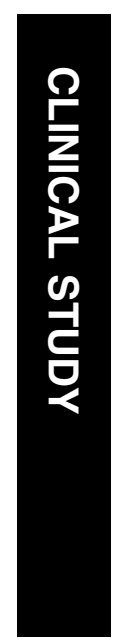

\section{A co-twin study of the relative effect of birth weight and gestational age on retinopathy of prematurity}

SJ Woo', KH Park' ${ }^{2}$ J Ahn', KJ Oh², SY Lee², EH Jeong ${ }^{2}$ and $\mathrm{KH}$ Park ${ }^{1}$
${ }^{1}$ Department of

Ophthalmology, Seoul National University College of Medicine, Seoul National University Bundang Hospital, Seongnam, Korea

${ }^{2}$ Department of Obstetrics and Gynecology, Seoul National University College of Medicine, Seoul National University Bundang Hospital, Seongnam, Korea

Correspondence: KH Park, Department of Obstetrics and Gynecology, Seoul National University College of Medicine, Seoul National University Bundang Hospital, 300 Gumi-dong, Bundang-gu, Seongnam-si, Gyeonggi-do, 463-707, Korea

Tel: + 82317877252 ;

Fax: + 82317874054

E-mail: pkh0419@snubh.org

Received: 19 January 2011 Accepted in revised form: 15 July 2011

Published online: 26 August 2011

\begin{abstract}
Purpose To determine the relative effect of birth weight and gestational age on retinopathy of prematurity (ROP) using preterm twin pairs discordant for birth weight. Methods This study was a retrospective cohort study including 55 consecutive twin pairs of $\mathbf{1 1 0}$ preterm infants (gestational age $\leq 33$ weeks). The outcomes of ROP including occurrence (any stage), severe ROP (stage 3 or more), and clinically significant ROP requiring laser treatment were compared between twins with the lower birth weight from each pair and their co-twins with the higher birth weight. Using twin pairs having different birth weight and identical gestational age, the independent effects of prematurity and intrauterine growth on ROP could be evaluated. Other perinatal morbidities related to prematurity were also compared between twin pairs.

Results No significant differences in ROP between larger and smaller infants were observed in the twin-paired analysis while analysis on individual infants showed strong association between small birth weight and ROP outcomes. However, in both the larger and smaller infant groups, gestational age of $<28$ weeks was significantly associated with ROP outcomes. No differences were found between twin pairs regarding other perinatal morbidities including bronchopulmonary dysplasia, respiratory distress syndrome, patent ductus arteriosus, intraventricular hemorrhage, and periventricular leukomalacia.

Conclusions Birth weight is not associated with ROP, while gestational age is in the twin-paired study, suggesting that gestational age is a better predictor of ROP than birth weight. This indicates that maturity is more
\end{abstract}

important in the pathogenesis of ROP than intrauterine growth.

Eye (2011) 25, 1478-1483; doi:10.1038/eye.2011.208; published online 26 August 2011

Keywords: retinopathy of prematurity; gestational age; birth weight

\section{Introduction}

Retinopathy of prematurity (ROP) is a common blinding disease in children and a major cause of vision loss among preterm infants. ${ }^{1}$ As survival of the very preterm infant has increased in recent years, the incidence of severe ROP, which is associated with vision loss, is reported to be increasing. ${ }^{2-4}$ Among factors suggested to have association with ROP, low gestational age and low birth weight are the strongest and most consistently identified risk factors. ${ }^{1,5,6}$ As gestational age is a major contributor of birth weight and these two factors closely correlate, the respective causal relationship in the pathogenesis of ROP and the superiority between the two factors are unclear. Some of the reported association between birth weight and ROP may have been influenced by this correlation between them. However, gestational age and birth weight mean different aspects of fetal health state: maturity and growth. Evaluating the independent effects of two factors on ROP is practically impossible in the usual settings of clinical study in preterm infants.

There is additional variability in birth weight that is unexplained by gestational age. This second type of birth weight is sometimes assumed to be a quality of growth and is strongly related to infant survival. ${ }^{7}$ In studies on preterm infants, ${ }^{6,8-11}$ the relative weight for 
gestational age (small-for-gestational age (SGA)) was shown to be a risk factor for severe ROP. However, another recent study ${ }^{12}$ has failed to prove the association between relative weight for gestational age (SGA) and ROP. The SGA infants are often exposed to different perinatal environments compared with AGA (appropriatefor-gestational age) infants including oxygen therapy and blood transfusion, and thus many factors are involved in the relationship between relative weight and ROP. To find the independent effect of birth weight on ROP, previous studies used statistical techniques to exclude the interactions of ROP with various clinical factors but could not completely escape the inter-correlations among the variables, which exist biologically.

As twins are exposed to an identical intrauterine environment for an equal period of time (gestational age), we may expect that the exact effect of birth weight on ROP could be determined by a twin study independent of other prenatal factors such as gestational age, intrauterine inflammation, and drug exposure.

The purpose of this study was to determine the relative effect of birth weight and gestational age on ROP. To evaluate the influences of birth weight on ROP independent of gestational age, we used a twin-paired analysis including high-risk preterm twin pairs discordant for birth weight, where other factors that influence birth weight such as gestational age can be controlled. We believe this is the sole method to separate the two related factors of birth weight and gestational age biologically, and to determine the influence of intrauterine growth on ROP.

\section{Materials and methods}

This was a retrospective cohort study of twin deliveries at Seoul National University Bundang Hospital (Seongnam, Korea), from July 2003 to November 2009. A detailed database of all obstetric patients and their neonates admitted to the neonatal intensive care unit at our institution has been constructed since 2003. From this clinical database, patients were retrospectively considered for inclusion in our analysis. The inclusion criteria were as follows: (1) twin pairs born at $\leq 33$ weeks of gestation; (2) living discordant twin pairs both of whom survived until 36 weeks post-menstrual age; and (3) twin pairs undergoing ROP screening examinations. Twin pairs with the same birth weight, or with one or both members being stillborn, or having major congenital malformation were excluded. Gestational age was calculated as the number of completed weeks of pregnancy, based on the last menstrual period or first or second trimester ultrasound. Medical records regarding ROP of the included preterm infants were reviewed by a single retina specialist (SJW) for the presence and stage of
ROP, laser treatment, and final outcome. The study was approved by the Institutional Review Board of the Seoul National University Bundang Hospital.

The data retrieved in the database included demographic information of the parents; placental pathology (chorionicity); neonatal characteristics (gestational age at birth, birth weight, gender, 5-min Apgar score, postnatal steroid therapy, blood transfusion, oxygen therapy, and mechanical ventilation); and neonatal morbidities (sepsis, bronchopulmonary dysplasia (BPD), respiratory distress syndrome (RDS), patent ductus arteriosus (PDA), intraventricular hemorrhage (IVH), periventricular leukomalacia (PVL), and necrotizing enterocolitis (NEC)). Chorionicity was assessed via post-delivery placental pathology and was available in all cases.

The screening examination for ROP followed the guidelines proposed by the American Academy of Ophthalmology and Pediatrics and the Association for Pediatric Ophthalmology and Strabismus. The first examination was performed 4-6 weeks from birth or 31-33 weeks post-conceptional age, whichever was later. The subsequent follow-up examination and laser treatment for type 1 ROP followed the clinical algorithm suggested by the Early Treatment for Retinopathy of Prematurity study results. ${ }^{13}$ The stage of ROP was defined as the highest stage during follow-up fundus examinations. Severe ROP was defined as stage 3,4 , or 5 .

The main outcome measures of ROP were the occurrence of any stage of ROP, severe ROP (stage 3 or more), and clinically significant ROP requiring laser treatment. Relative effects of birth weight and gestational age on ROP were examined as both a continuous variable and a categorical predictor according to cutoff values. Birth weight was categorized as $\leq 1000 \mathrm{~g}$ and $>1000 \mathrm{~g}$; and gestational age was categorized as $\leq 28$ weeks and $>28$ weeks. Twins were considered as either individuals or as members of twin pairs, depending on the analyses. We first examined the associations between gestational age, birth weight, and ROP on individual twin data. To evaluate the effect of birth weight on ROP, independent of other factors that influence birth weight such as gestational age, within-pair analysis was subsequently conducted: we compared ROP outcomes of twins with lower birth weight with those of their co-twins with higher birth weight. Finally, to evaluate the influence of gestational age on ROP, we divided the twin pairs into two subgroups based on birth weight in each pair, namely a larger and a smaller subgroup, and then performed an analysis separately in each subgroup.

Shapiro-Wilk and Kolmogorov-Smirnov tests were used to affirm normal distribution of the data. Univariate analysis was conducted for unpaired data with the Student $t$-test, Mann--Whitney $U$-test, $\chi^{2}$-test, or Fisher's 
exact test. A Pearson correlation analysis was used to evaluate the correlation between the gestational age and birth weight. Univariate paired comparisons on dichotomous outcomes between the larger infant from the twin pair and the smaller infant from the same pair were performed using the McNemar test and paired $t$-test. All reported $P$-values are two-sided, and $P$-values $<0.05$ were considered statistically significant.

Continuous variables were expressed as mean \pm SD.

Statistical analyses were performed using SPSS for Windows (version 17.0; Statistical Package for the Social Sciences, SPSS, Inc., Chicago, IL, USA).

\section{Results}

During the study period, a total of 700 twin pairs were born at our tertiary care center. Eighty-eight twin pairs $(12.6 \%)$ were born at $\leq 33$ weeks gestation. Of these 88 twin pairs, 31 twin pairs were excluded because one or both twins had died in the delivery room $(n=8)$, had died before ophthalmologic screening for ROP $(n=10)$, had had major congenital malformations $(n=3)$, and were lost to follow-up $(n=10)$, and 2 twin pairs were excluded because the birth weight of the twins within a pair was equal. The remaining 55 twin pairs were included in the final analysis. There were $41(74.5 \%)$ dichorionic twins and $14(25.5 \%)$ monochorionic twins. The mean gestational age of the cohort was $29.9 \pm 2.3$ weeks (range, 25.1-33.0 weeks), and the mean birth weight was $1340 \pm 388 \mathrm{~g}$ (range, 615-2420 g). ROP was diagnosed in 39 of 110 infants $(35.5 \%)$ from our cohort, including 22 with mild ROP (20.0\%: stage 1, $n=10$; stage $2, n=12)$ and 17 with severe ROP (15.5\%, stage 3$)$. There were no cases with stage 4 or 5 ROP. Laser treatment was performed on 12 infants (10.9\%).

Based on the univariate analyses of the total infants, as expected, gestational age and birth weight were significantly associated with the occurrence and progression of ROP, as well as laser treatment. Mean gestational age was significantly earlier in the infant group showing occurrence of any stage of ROP (28.3 vs 30.6 weeks, $P<0.0001$ ), stage 3 ROP (26.6 vs 30.4 weeks, $P<0.0001$ ), and laser treatment (26.7 vs 30.2 weeks, $P<0.0001$ ) (Figure 1). Birth weight was also significantly smaller in the infant group showing occurrence of any stage of ROP (1089 vs $1479 \mathrm{~g}, P<0.0001$ ), stage 3 ROP (938 vs 1414 g, $P<0.0001$ ), and laser treatment (920 vs $1392 \mathrm{~g}, P<0.0001)$. As gestational age and birth weight were highly correlated $(r=0.815, P<0.0001)$, the two variables could not be assumed to be independently predictive of the occurrence and progression of ROP. Therefore, the withinpair analysis was conducted to determine the effect of birth weight on ROP, independent of other factors that influence birth weight including gestational age.
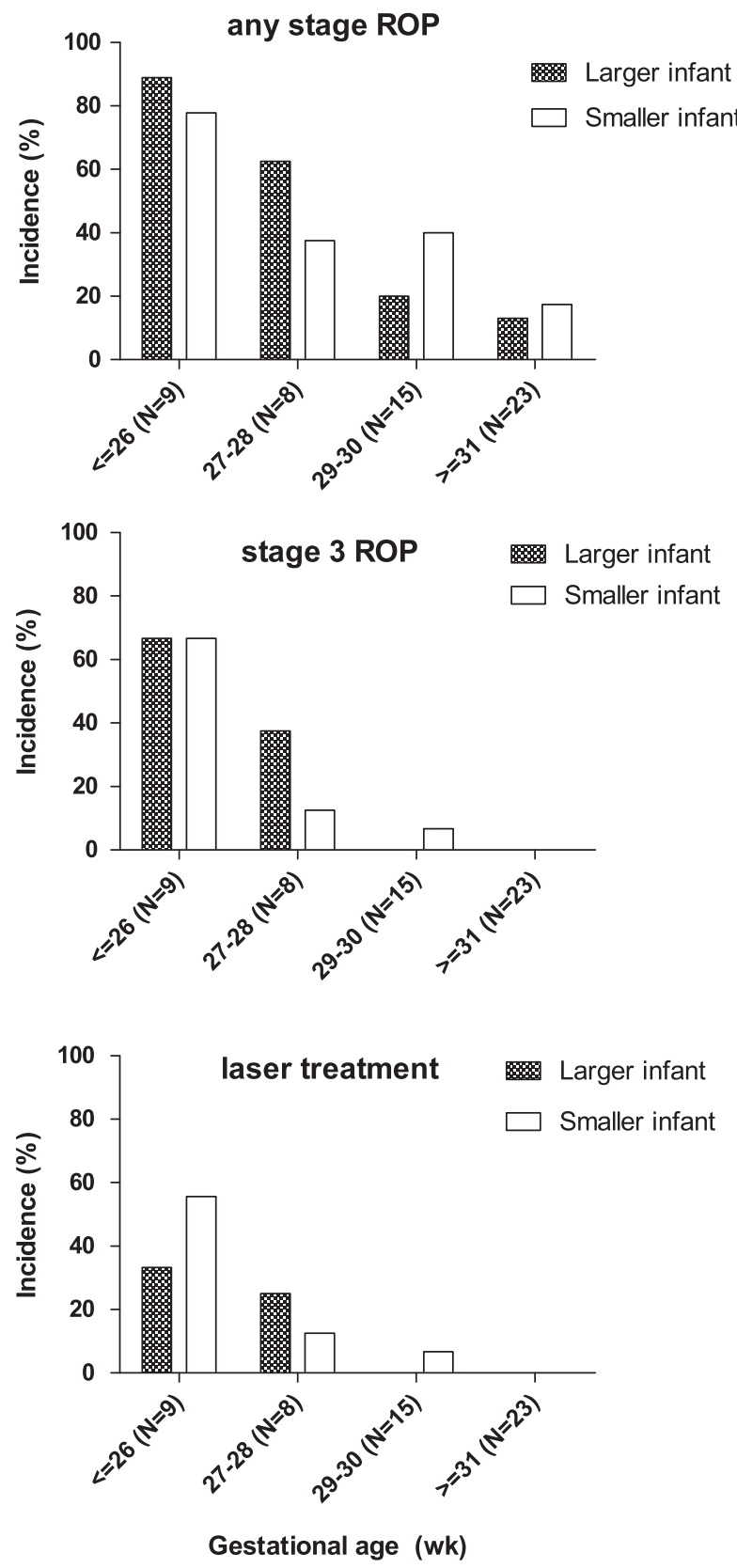

Figure 1 The incidences of any stage of ROP, stage 3 ROP, and laser treatment, which were plotted against the gestational age. There were no significant differences of occurrence and progression of ROP between smaller and larger twin infants.

Results of paired comparisons on clinical features and perinatal outcomes according to the birth weight of infants within twin pairs are shown in Table 1. The mean difference in birth weight between the two groups was $206 \mathrm{~g}(P<0.001)$, and the mean birth weight discordance within twin pairs was $13.5 \%$. The proportion of infants with a birth weight $\leq 1000 \mathrm{~g}$ was significantly lower in the larger twin group than in the smaller twin group 
(16.4 vs $30.9 \%, P=0.008$ ). There were no differences in the occurrence and progression of ROP and laser treatment between larger and smaller infants (any stage of ROP, 34.5 vs $36.4 \%, P=1.000$; stage 3 ROP, 16.4 vs $14.5 \%, P=1.000$; laser treatment, 9.1 vs $12.7 \%, P=0.727$ by McNemar test, Figure 1 and Table 1). Furthermore, the incidence of other perinatal morbidities including BPD, RDS, PDA, IVH, PVL, NEC, and sepsis was not different between the smaller and larger twins. Gender

distribution and all other clinical characteristics, such as Apgar score $<7$ at 5 minutes, postnatal steroid treatment, blood transfusion, supplemental oxygen therapy, and

Table 1 Paired comparisons of clinical features and perinatal outcomes between larger and smaller infants of 55 twin pairs

\begin{tabular}{|c|c|c|c|}
\hline & $\begin{array}{l}\text { Larger } \\
\text { infant } \\
(\mathrm{N}=55)\end{array}$ & $\begin{array}{l}\text { Smaller } \\
\text { infant } \\
(\mathrm{N}=55)\end{array}$ & $\mathrm{P}$ \\
\hline Birth weight ( $g$, mean $\pm S D$ ) & $1444 \pm 388$ & $1238 \pm 363$ & $<0.001$ \\
\hline Birth weight $\leq 1000 \mathrm{~g}$ & $9(16.4 \%)$ & $17(30.9 \%)$ & 0.008 \\
\hline \multicolumn{4}{|l|}{ ROP outcomes } \\
\hline Any stage ROP & $19(34.5 \%)$ & $20(36.4 \%)$ & 1.000 \\
\hline Stage 3 ROP & $9(16.4 \%)$ & $8(14.5 \%)$ & 1.000 \\
\hline Laser treatment & $5(9.1 \%)$ & $7(12.7 \%)$ & 0.727 \\
\hline \multicolumn{4}{|l|}{ Perinatal morbidities } \\
\hline Bronchopulmonary dysplasia & $19(34.5 \%)$ & $21(38.2 \%)$ & 0.625 \\
\hline Respiratory distress syndrome & $33(60.0 \%)$ & $27(49.1 \%)$ & 0.238 \\
\hline Patent ductus arteriosus & $28(50.9 \%)$ & $29(52.7 \%)$ & 1.000 \\
\hline $\begin{array}{l}\text { Intraventricular hemorrhage } \\
\text { (grade } 2 \text { or more) }\end{array}$ & $2(3.6 \%)$ & $3(5.5 \%)$ & 1.000 \\
\hline Periventricular leukomalacia & $7(12.7 \%)$ & $5(9.1 \%)$ & 0.727 \\
\hline Necrotizing enterocolitis & $0(0.0 \%)$ & $0(0.0 \%)$ & 1.000 \\
\hline Sepsis & $5(9.1 \%)$ & $11(20.0 \%)$ & 0.070 \\
\hline \multicolumn{4}{|l|}{ Clinical features } \\
\hline Female infant & $23(41.8 \%)$ & $29(52.7 \%)$ & 0.286 \\
\hline 5-min Apgar score $<7$ & $13(23.6 \%)$ & $21(38.2 \%)$ & 0.096 \\
\hline Dexamethasone treatment & $48(87.3 \%)$ & $48(87.3 \%)$ & 1.000 \\
\hline Blood transfusion & $27(49.1 \%)$ & $32(58.2 \%)$ & 0.125 \\
\hline Oxygen therapy & $42(76.4 \%)$ & $42(76.4 \%)$ & 1.000 \\
\hline Mechanical ventilation & $37(67.3 \%)$ & $31(56.4 \%)$ & 0.210 \\
\hline
\end{tabular}

Abbreviation: ROP, retinopathy of prematurity.

$P$-values were calculated using paired $t$-test and McNemar test. mechanical ventilation, were not significantly different between the two groups. Additional analyses of ROP outcomes in subgroups according to the chorionicity and discordance yielded similar results showing no difference between smaller and larger twin infants.

As a next step, we evaluated the influence of gestational age on ROP in each of two subgroups on the basis of birth weight in each twin pair (ie, a larger and a smaller subgroup) (Table 2). In a subgroup of the larger twin infants at birth, the proportion of infants with a birth weight $\leq 1000 \mathrm{~g}$ was significantly higher in infants born at $\leq 28$ weeks $(52.9 \%, 9 / 17)$ than in infants born at $>28$ weeks $(0.0 \%, 0 / 38)$. In contrast to twin-paired analysis, infants born at $\leq 28$ weeks had significantly higher rates of ROP occurrence and severity and ROP requiring laser treatment $(76.5,52.9$, and $29.4 \%)$ than those born at $>28$ weeks $(15.8,0.0$, and $0.0 \% ; P<0.005)$. In a subgroup of the smaller twin infants at birth, the proportion of infants with a birth weight $\leq 1000 \mathrm{~g}$ was significantly higher in infants born at $\leq 28$ weeks $(70.6 \%$, $12 / 17)$ than in infants born at $>28$ weeks $(13.2 \%, 5 / 38)$. Similar results were also obtained in a subgroup of the smaller twin infants regarding ROP outcomes (any-stage ROP:stage 3 ROP:laser treatment $=58.8 \%: 41.2 \%: 35.3 \%$ (gestational age $\leq 28$ weeks) vs $26.3 \%: 2.6 \%: 2.6 \%$ (gestational age $>28$ weeks), $P<0.05$ ).

Unpaired comparisons of ROP outcomes and perinatal morbidities showed no significant differences between SGA (birth weight $<10$ th percentile of normal twin population) and AGA (birth weight $\geq 10$ th percentile) infants categorized from our twin infants. Only the incidence of sepsis was slightly higher in the SGA infant group than the AGA group in our subgroup analysis without statistical significance (26.1 vs $11.5 \%, P=0.098$ by $\chi^{2}$-test).

\section{Discussion}

Comparative analyses on twin pairs with different birth weight showed that birth weight per se is not associated

Table 2 The influence of gestational age on retinopathy of prematurity (ROP) within either larger or smaller infants of 55 twin pairs

\begin{tabular}{|c|c|c|c|c|c|c|}
\hline & \multicolumn{3}{|c|}{ Larger infant $(\mathrm{N}=55)$} & \multicolumn{3}{|c|}{ Smaller infant $(\mathrm{N}=55)$} \\
\hline & $\begin{array}{c}G A \leq 28 \text { weeks } \\
(\mathrm{N}=17)\end{array}$ & $\begin{array}{c}G A>28 \text { weeks } \\
(\mathrm{N}=38)\end{array}$ & $\mathrm{P}$ & $\begin{array}{c}G A \leq 28 \text { weeks } \\
(\mathrm{N}=17)\end{array}$ & $\begin{array}{c}G A>28 \text { weeks } \\
(\mathrm{N}=38)\end{array}$ & $\mathrm{P}$ \\
\hline Birth weight $(g$, mean $\pm S D$ ) & $1007 \pm 162$ & $1639 \pm 285$ & $<0.001$ & $909 \pm 125$ & $1384 \pm 337$ & $<0.001$ \\
\hline Birth weight $\leq 1000 \mathrm{~g}$ & $9(52.9 \%)$ & $0(0.0 \%)$ & $<0.001$ & $12(70.6 \%)$ & $5(13.2 \%)$ & $<0.001$ \\
\hline Any stage ROP & $13(76.5 \%)$ & $6(15.8 \%)$ & $<0.001$ & $10(58.8 \%)$ & $10(26.3 \%)$ & 0.021 \\
\hline Stage 3 ROP & $9(52.9 \%)$ & $0(0.0 \%)$ & $<0.001$ & $7(41.2 \%)$ & $1(2.6 \%)$ & 0.001 \\
\hline Laser treatment & $5(29.4 \%)$ & $0(0.0 \%)$ & 0.002 & $6(35.3 \%)$ & $1(2.6 \%)$ & 0.002 \\
\hline
\end{tabular}

Abbreviations: GA, gestational age; ROP, retinopathy of prematurity.

$P$-values were calculated using Mann-Whitney $U$-test, $\chi^{2}$-test, and Fisher's exact test. 
with the occurrence or severity of ROP while gestational age is. The lack of difference between smaller and larger twin infants was also reported previously in BPD, which is known to have a similar pathophysiology to ROP. ${ }^{14}$

Most studies ${ }^{6,8-11}$ comparing the incidence of ROP in SGA and AGA infants reported similar conclusions that SGA infants have a higher risk of developing any stage of ROP or severe ROP. Darlow et $a l^{6}$ showed that the relative birth weight for gestational age was associated with ROP in a dose-response manner independent of gestational age. Bardin et $a l^{10}$ reported that SGA infants have an increased risk of developing any stage of ROP (90 vs 58\%) and severe ROP (65 vs 12\%). Gortner et al ${ }^{9}$ reported that SGA infants have an increased risk of ROP than AGA infants (37 vs 15\%), as well as increased risk of developing chronic lung disease and prolonged oxygen therapy, while there was no difference in stage 3 ROP between SGA and AGA groups. Allegaert et $a l^{11}$ reported that SGA infants have 3.7-fold higher risk of developing threshold ROP than AGA infants. In contrast, Dhaliwal et $a l^{8}$ reported that there were no differences in the incidence of severe ROP requiring laser treatment between SGA and AGA infants, although SGA infants were more likely to develop any stage of ROP and severe ROP than their AGA peers. Moreover, among infants with gestational age earlier than 26 weeks, they found no difference in the incidence of any stage of ROP or severe ROP. Furthermore, a recent study ${ }^{12}$ on 345 high-risk infants (birth weight $\leq 1500 \mathrm{~g}$ and gestational age $\leq 32$ weeks) reached a conclusion that being SGA was not a significant risk factor for any stage of ROP or for severe ROP. Therefore, the difference between ROP outcomes between SGA and AGA infants is still confusing and has yet to be determined. The reason for these conflicting results may be due to different cohorts of preterm infants with variable general state and morbidity. Another reason could be the complicated interactions between ROP and many perinatal factors, such as oxygen therapy, perinatal inflammation, and blood transfusion.

Compared with the previous studies, the strength of our study was that by using twin-pair comparisons, most prenatal factors related to the development of ROP could be made equivalent between the two groups with different birth weight. Therefore, the true influence of birth weight or intrauterine growth on ROP could be determined. Moreover, the independent effect of birth weight on other perinatal morbidities could also be evaluated in this twin-paired study.

Of note is that the incidence of sepsis was twice more frequent in smaller infants, and that blood transfusion was more frequently required in smaller infants although the difference did not reach the statistical significance. Additionally, incidence of sepsis was also slightly higher in the SGA infant group than the AGA group in our subgroup analysis. These results could explain the known conception ${ }^{7}$ that SGA is associated with increased perinatal mortality. Although intrauterine growth restriction was not directly associated with ROP in our study, it might be associated with perinatal mortality through systemic inflammation or sepsis.

There are limitations in our study. First, the preterm twin cohort is the minority group among general preterm infants and has limitations in generalizing this result to the population. A second limitation is the small number of infants included in our study. We could not find any significant differences in the incidence of any stage of ROP or severe ROP between twin pairs, but the very small differences in the incidence of ROP between the two groups may be revealed to be significant in future studies including a larger number of preterm twins. Third, the birth weight discordance of twin pairs was not severe as the mean birth weight discordance was $13.5 \%$ and as the number of significantly discordant twins (discordance $\geq 15 \%$ ) was 16 pairs out of 55 pairs (29.1\%). Although analysis on the subgroup of discordant twins did not yield significant changes in our result, additional research including larger number of twin infants with extreme discordance is required in future.

In conclusion, birth weight per se is not associated with any stage of ROP or severe ROP in preterm twins. This indicates that maturity is more important in the pathogenesis of ROP than intrauterine growth.

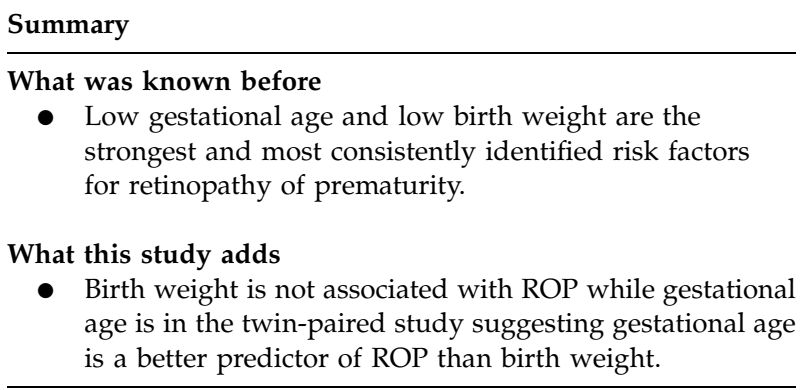

\section{Conflict of interest}

The authors declare no conflict of interest.

\section{References}

1 Chen J, Smith LE. Retinopathy of prematurity. Angiogenesis 2007; 10: 133-140.

2 Todd DA, Wright A, Smith J. Severe retinopathy of prematurity in infants $<30$ weeks' gestation in New South Wales and the Australian Capital Territory from 1992 to 2002. Arch Dis Child Fetal Neonatal Ed 2007; 92: F251-F254.

3 Todd DA, Kennedy J, Cassell C, Roberts S, John E. Retinopathy of prematurity in infants $<29$ weeks' gestation 
at birth in New South Wales from 1986-92. J Paediatr Child Health 1998; 34: 32-36.

4 Allegaert K, de Coen K, Devlieger H. Threshold retinopathy at threshold of viability: the EpiBel study. Br J Ophthalmol 2004; 88: 239-242.

5 McColm JR, Fleck BW. Retinopathy of prematurity: causation. Semin Neonatol 2001; 6: 453-460.

6 Darlow BA, Hutchinson JL, Henderson-Smart DJ, Donoghue DA, Simpson JM, Evans NJ. Prenatal risk factors for severe retinopathy of prematurity among very preterm infants of the Australian and New Zealand Neonatal Network. Pediatrics 2005; 115: 990-996.

7 Wilcox AJ. Birth weight and perinatal mortality: the effect of maternal smoking. Am J Epidemiol 1993; 137: 1098-1104.

8 Dhaliwal CA, Fleck BW, Wright E, Graham C, McIntosh N. Retinopathy of prematurity in small-for-gestational age infants compared with those of appropriate size for gestational age. Arch Dis Child Fetal Neonatal Ed 2009; 94: F193-F195.

9 Gortner L, Wauer RR, Stock GJ, Reiter HL, Reiss I, Jorch G et al. Neonatal outcome in small for gestational age infants: do they really better? J Perinat Med 1999; 27: 484-489.
10 Bardin C, Zelkowitz P, Papageorgiou A. Outcome of smallfor-gestational age and appropriate-for-gestational age infants born before 27 weeks of gestation. Pediatrics 1997; 100: E4.

11 Allegaert K, Vanhole C, Casteels I, Naulaers G, Debeer A, Cossey V et al. Perinatal growth characteristics and associated risk of developing threshold retinopathy of prematurity. J AAPOS 2003; 7: 34-37.

12 Fortes Filho JB, Valiatti FB, Eckert GU, Costa MC, Silveira $\mathrm{RC}$, Procianoy RS. Is being small for gestational age a risk factor for retinopathy of prematurity? A study with 345 very low birth weight preterm infants. J Pediatr (Rio J) 2009; 85: 48-54.

13 Early Treatment For Retinopathy Of Prematurity Cooperative G. Revised indications for the treatment of retinopathy of prematurity: results of the early treatment for retinopathy of prematurity randomized trial. Arch Ophthalmol 2003; 121: 1684-1694.

14 Capoluongo E, Ameglio F, Zuppi C. Insulin-like growth factor-I and complications of prematurity: a focus on bronchopulmonary dysplasia. Clin Chem Lab Med 2008; 46: 1061-1066. 